\title{
Decreased Tongue Strength is Related to Skeletal Muscle Mass in COPD Patients
}

\author{
Ryuji Sugiya ${ }^{1,2}\left(\right.$ Puji Higashimoto $^{1} \cdot$ Masashi Shiraishi ${ }^{1} \cdot$ Tomomi Tamura $^{1} \cdot$ Tamotsu Kimura $^{1} \cdot$ Yasutaka Chiba $^{3}$. \\ Osamu Nishiyama ${ }^{4} \cdot$ Shinichi Arizono ${ }^{2} \cdot$ Kanji Fukuda $^{1} \cdot$ Yuji Tohda $^{4}$
}

Received: 6 November 2020 / Accepted: 6 May 2021 / Published online: 26 May 2021

(c) The Author(s), under exclusive licence to Springer Science+Business Media, LLC, part of Springer Nature 2021

\begin{abstract}
Dysphagia is frequently observed in patients with chronic obstructive pulmonary disease (COPD). Decreased tongue strength is one of the causes of dysphagia, and it is often observed in patients with sarcopenia. Sarcopenia is also frequently observed in COPD patients. We hypothesized that tongue strength is lower in COPD patients compared to normal subjects. This was a single-center, observational, cross-sectional study. Maximum tongue pressure (MTP) was measured in 27 patients with COPD and 24 age-matched control subjects. We also evaluated handgrip strength, gait speed, and appendicular skeletal muscle mass to define subjects as having sarcopenia. We used bioelectrical impedance analysis to assess body composition. The eating assessment test-10 was used to diagnose dysphagia. MTP was significantly lower in COPD patients than in control subjects $(33.8 \pm 8.4$ vs $38.0 \pm 5.3 ; p=0.032)$. All measures of muscle and fat free body mass, handgrip strength, and gait speed were also significantly lower in COPD patients compared to control subjects $(p<0.01)$. The prevalence of sarcopenia in COPD patients was higher than that in control subjects $(6 / 27$ versus $0 / 24 ; p=0.007)$, but the prevalence of dysphagia was not different between groups (COPD: $5 / 27$, versus control: $1 / 24 ; p=0.112$ ). MTP was moderately correlated with skeletal muscle mass index $(r=0.56, p=0.003)$ and handgrip strength $(r=0.43, p=0.027)$ in COPD patients. Tongue strength was lower in COPD patients compared to normal subjects, and decreased tongue strength may be correlated with sarcopenia in COPD patients.
\end{abstract}

Keywords Chronic obstructive pulmonary disease $\cdot$ Tongue strength $\cdot$ Skeletal muscle mass $\cdot$ Handgrip strength $\cdot$ Gait speed

Ryuji Sugiya

ryuji.sugiya@med.kindai.ac.jp

1 Department of Rehabilitation Medicine, Kindai University Faculty of Medicine, 377-2 Ohnohigashi, Osakasayama, Osaka 5898511, Japan

2 School of Rehabilitation Sciences, Seirei Christopher University, 3453 Mikatahara, Hamamatsu, Shizuoka 4338558, Japan

3 Clinical Research Center, Kindai University hospital, 377-2 Ohnohigashi, Osakasayama, Osaka 5898511, Japan

4 Department of Respiratory Medicine and Allergology, Kindai University Faculty of Medicine, 377-2 Ohnohigashi, Osakasayama, Osaka 5898511, Japan

\section{Introduction}

Dysphagia is a common complication for patients with chronic obstructive pulmonary disease (COPD), seen in about $85 \%$ of COPD patients [1]. Dysphagia in COPD patients is caused by decreased swallowing function due to various causes. Decreased tongue strength strongly affects dysphagia in patients with Parkinson's disease and cerebrovascular disease. Tongue strength is a part of the swallowing function [2]. The tongue plays a major role in bolus formation, control, and propulsion in swallowing [3]. In the preparation and oral phase of swallowing, a bolus is formed and is placed by the tongue on the occlusal surface of the posterior teeth and buccal mucosa for transport to the pharynx. When the tongue transfers the bolus to the pharyngeal cavity, it must raise the oral pressure to prevent pharyngeal residue. Decreased tongue strength causes pharyngeal residue, and it may lead to dysphagia. Therefore, evaluation of tongue 
strength is necessary in determining swallowing function. Tongue strength will decline in patients not only from the effects of disease such as stroke and amyotrophic lateral sclerosis [4, 5], but also from sarcopenia.

Sarcopenia is characterized by low muscle strength and low muscle mass [6]. In COPD patients, being underweight is associated with higher mortality [7]. Sarcopenia is likely to occur in COPD patients due to the effects of systematic inflammation, oxidative stress, smoking, hypoxemia, chronic inactivity, and malnutrition [8]. It may cause not only wholebody atrophy but also weakness of the tongue. Quadriceps muscle and handgrip strength have been evaluated to assess muscle weakness in COPD patients $[9,10]$. However, tongue strength in COPD patients has not been investigated yet. Decreased tongue strength may be a contributing factor to dysphagia in patients with COPD.

The relationship between tongue strength and sarcopenia has been reported in previous studies [11, 12]. One of the ways to assess tongue strength is to measure maximum tongue pressure (MTP). MTP is associated with age, serum albumin concentration, activities of daily living, and the presence of sarcopenia [13]. In patients with sarcopenic dysphagia, atrophy of the swallowing muscles has been observed by the thickness and brightness of tongue muscles using ultrasonography [14]. MTP is also decreased in frail elderly persons [15].

We hypothesized that MTP would be lower in COPD patients compared to normal subjects.

\section{Materials and Methods}

\section{Study Subjects}

This was a single-center, observational, cross-sectional study. Twenty-seven elderly patients ( $>65$ years old) with stable COPD were recruited for enrollment in this study. They were chosen from a group of patients who had visited the Department of Respiratory Medicine and Allergology at Kindai University Hospital from December 2019 to May 2020. We also recruited 24 age-matched control subjects who did not have any pulmonary disease. Exclusion criteria included metallic implants and a history of stroke or neuromuscular disease. Subjects who did not have their front teeth were excluded, because they were not be able to bite down on the equipment used to measure MTP. According to a preliminary study, the mean MTP in healthy subjects (age $\geq 70$ ) was $31.9 \pm 8.9 \mathrm{kPa}$ [16]. In the COPD patients, we assumed $25.5 \pm 7.7 \mathrm{kPa}$ for MTP by referring to the data of hospitalized patients [13]. With these values, the required number of cases would be 54 (27 subjects in each group) based on Student's $t$-test, which was used to assess the difference between the MTP value of the 2 groups, with a significance level of 5\% (both sides) and a study power of $80 \%$. Twenty-seven participants per group were recruited. However, three subjects in the control group were dropped because of erroneous values in their BIA measurements. They were not replaced because the coronavirus had spread in Japan, and we could not recruit anymore subjects. Thus, we had 51 subjects (27 COPD patients and 24 age-matched controls) for the final study.

All subjects included in the study had undergone spirometry (CHEST AC-55V, Chest, Tokyo, Japan) within the last 3 months, according to ATS guidelines [17]. COPD patients were diagnosed using the Global Initiative lung Disease (GOLD) criteria [18].

Protocols were approved by the Committee for Ethics at Kindai University School of Medicine before study enrollment, and all subjects signed informed consents (No. 31-116).

\section{Assessment of Tongue Strength}

We measured maximum tongue pressure (MTP) using a balloon-based tongue pressure measurement device (Shown in Fig. 1, JMS tongue pressure measuring device ${ }^{\circ}$, JMS, Hiroshima, JAPAN) according to the methods proposed by Takahashi [19]. The probe was inflated at a baseline pressure of 19.6 kilopascal $(\mathrm{kPa})$. To measure MTP, the balloon

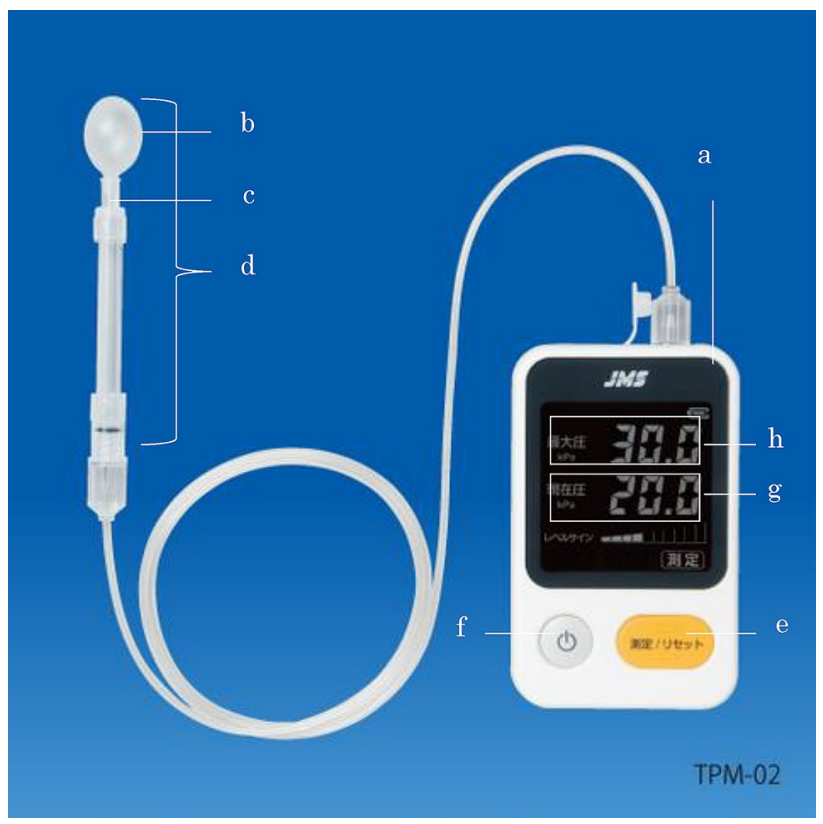

Fig. 1 A balloon-based tongue pressure measurement device (JMS tongue pressure measurement devise $\AA$ ). (a) Digital tongue pressure measurement device; (b) balloon (width: $18 \mathrm{mmm}$, height: $25 \mathrm{~mm}$ ); (c) plastic pipe (width: $6 \mathrm{~mm}$, height: $10 \mathrm{~mm}$ ); (d) disposable probe; (e) measurement/reset; (f) power; (g) present pressure; (h) maximum pressure 
was positioned on the anterior palate with the lips closed. Subjects raised their tongue and compressed the balloon onto the palate with maximal voluntary muscular effort for approximately $7 \mathrm{~s}$. The measurement was performed three times with a 1-min interval rest between trials. The maximum value was recorded as the MTP.

\section{Assessment of Sarcopenia}

The Asian Working Group for Sarcopenia (AWGS) 2019 consensus established the criteria for sarcopenia in Asian people [20] (Supplementary Fig. 1). Sarcopenia is diagnosed using appendicular skeletal muscle mass (ASM), handgrip strength, and gait speed. The following cut-off values are used to identify sarcopenia: ASM of $<7.0 \mathrm{~kg} / \mathrm{m}^{2}$ for men and $<5.7 \mathrm{~kg} / \mathrm{m}^{2}$ for women, and either a handgrip strength of $<28 \mathrm{~kg}$ for men and $<18 \mathrm{~kg}$ for women or a gait speed of $1.0 \mathrm{~m} / \mathrm{s}$.

For diagnosing cases in a clinical research setting, the Strength, Assistance with walking, Rise from a chair, climb stairs, and Falls (SARC-F) questionnaire is recommended by the AWGS. This questionnaire consists of 5 simple questions and each item scores from 0 to 2 points. A total score of $\geq 4$ corresponds to the cut-off for sarcopenia. We evaluated the SARC-F only in the control subjects because subjects with chronic conditions (heart failure, COPD, diabetes mellitus, chronic kidney disease, etc.) can be exempt from this screening tool. The validity of the Japanese version of the SARC-F has been reported in a previous study [21].

Handgrip strength was measured three times for the right hand using a grip strength dynamometer GRIP-D (Takei Scientific Instruments, Niigata, Japan) and mean values were used. To assess gait speed, subjects were asked to walk a timed 6-m course at their usual speed. Time commenced when the subjects started foot movement and stopped when the foot contacted the ground after completely crossing the 6-m mark.

\section{Bioelectrical Impedance Analysis}

Body composition was measured using the seca mBCA525 (seca Nihon, Chiba, Japan). The seca mBCA525 is designed for measurements in the supine position, using four pairs of electrodes (eight electrodes in total), positioned on each hand and foot [22]. Supine measurements were taken after lying down for $10 \mathrm{~min}$. Alcohol was prohibited for $24 \mathrm{~h}$ prior to the impedance measurements. We adjusted for body size using height squared. Body mass index (BMI), fat free mass index (FFMI), fat mass index (FMI), appendicular skeletal muscle mass (ASM), and skeletal muscle mass index (SMI) were calculated.

\section{Assessment of Swallowing Function}

Swallowing function was assessed using the eating assessment test-10 (EAT-10) which indicates the subject's selfperception of swallowing function [23] (Supplementary table 1). The EAT-10 consists of 10 items. Each item includes a statement describing a scenario that could be a problem for a subject experiencing swallowing difficulty. Subjects scored their symptoms from 0 to 4 (0: no problem, 4: severe problem), with a total score of 3 or higher indicating dysphagia.

\section{Statistical Analysis}

Results are shown as the mean \pm standard deviation (SD). $p$ Values $<0.05$ were considered significant for all analyses. The differences between each measurement among COPD patients and control subjects were compared with a $t$-test and $\chi^{2}$ statistics. Differences in MTP between COPD and control groups and between males and females were analyzed by two-way analysis of variance. Differences between COPD stages (stage I + II, stage III, stage IV) were analyzed by ANOVA with repeated measures. The correlations between MTP and other factors were assessed using a Pearson correlation analysis. Analyses were carried out using SPSS24.0 (Tokyo, Japan).

\section{Results}

\section{Subject Characteristics}

The characteristics of subjects are summarized in Table 1 . Age and gender ratio were not different between groups ( $p=0.948, p=0.856$, respectively). Smoking history (pack years) was significantly higher in COPD patients compared to control subjects $(p<0.001)$. Prevalence of dyslipidemia was higher in COPD patients $(p=0.016)$. Four of the COPD patients, but none in the control group had chronic cardiovascular disease $(p=0.555)$.

\section{Comparison of MTP, Prevalence of Sarcopenia, and Dysphagia (Table 2)}

MTP was significantly lower in COPD patients than in control subjects $(33.6 \pm 8.4$ versus $38.0 \pm 5.3 \mathrm{kPa}$; $p=0.032$ ) (Fig. 2). There were significant differences between genders $(F(1,47)=4.664, p<0.036)$. MTP was lower among female than male COPD patients. The prevalence of sarcopenia was higher in COPD patients compared with control subjects (6/27 versus 0/24; $p=0.007$ ) (Table 2). ASM, FFMI, SMI, handgrip 
Table 1 Participant demographic characteristics

\begin{tabular}{lllr}
\hline & Control subjects $(n=24)$ & COPD patients $(n=27)$ & $p$ value \\
\hline Age (years) & $77.5 \pm 5.0$ & $77.6 \pm 4.6$ & 0.948 \\
Male, $n(\%)$ & $20(83)$ & $23(85)$ & 0.856 \\
Smoking history, $n(\%)$ & $13(54)$ & $24(89)$ & 0.014 \\
Smoking history & $18.5 \pm 24.9$ & $68.4 \pm 39.9$ & $<0.001$ \\
(pack years) & & & \\
FVC (L) & $3.2 \pm 0.7$ & $2.8 \pm 0.8$ & 0.092 \\
$\%$ FVC (\%) & $104.7 \pm 18.6$ & $90.6 \pm 20.6$ & 0.010 \\
FEV $(\mathrm{L})$ & $2.3 \pm 0.5$ & $1.3 \pm 0.6$ & $<0.001$ \\
$\%$ FEV $(\%)$ & $97.2 \pm 19.2$ & $50.4 \pm 21.1$ & $<0.001$ \\
GOLD stage, $n$ & - & $10 / 10 / 7$ & - \\
(I+II/III/I) & & & - \\
mMRC dyspnea scale & - & $0 / 4 / 17 / 6 / 0$ & \\
$(0 / 1 / 2 / 3 / 4)$ & & & 0.166 \\
Comorbidity, $n$ (\%) & & $13(48)$ & 0.016 \\
Hypertension & $7(29)$ & $10(42)$ & 0.200 \\
Dyslipidemia & $2(13)$ & $5(19)$ & 0.555 \\
Diabetes mellitus & $2(8)$ & $5(19)$ & - \\
Chronic cardiovascular disease & $3(13)$ & $0(0)$ & - \\
History of aspiration pneumonia, $n(\%)$ & $0(0)$ & $4(15)$ & \\
LTOT, $n$ (\%) & - & & \\
\hline
\end{tabular}

Mean \pm standard deviation

FVC forced vital capacity, FEVI forced expiratory volume in one second, mMRC dyspnea scale modified medical research council dyspnea scale, LTOT long term oxygen therapy, GOLD global initiative for chronic obstructive lung disease

Table 2 Measurement values in control subjects and COPD patients

\begin{tabular}{|c|c|c|c|c|c|}
\hline & \multirow[t]{2}{*}{$\begin{array}{l}\text { Control subjects } \\
(n=24)\end{array}$} & \multicolumn{4}{|l|}{$\begin{array}{l}\text { COPD Patients } \\
(n=27)\end{array}$} \\
\hline & & $\begin{array}{l}\text { All COPD patients } \\
(n=27)\end{array}$ & $\begin{array}{l}\text { GOLD stage I+ II } \\
(n=10)\end{array}$ & $\begin{array}{l}\text { GOLD stage III } \\
(n=10)\end{array}$ & $\begin{array}{l}\text { GOLD stage IV } \\
(n=7)\end{array}$ \\
\hline $\mathrm{MTP}(\mathrm{kPa})$ & $38.0 \pm 5.3$ & $33.6 \pm 8.4 *$ & $33.3 \pm 11.0$ & $34.2 \pm 5.5$ & $33.1 \pm 7.1$ \\
\hline Sarcopenia, $n(\%)$ & $0(0)$ & $6(22)^{* *}$ & $1(10)$ & $4(40)$ & $2(28)$ \\
\hline SARC-F abnormal $(n)$ & 0 & - & - & - & - \\
\hline Handgrip strength (kg) & $36.0 \pm 8.4$ & $29.5 \pm 7.5^{* *}$ & $31.6 \pm 7.5$ & $28.4 \pm 6.2$ & $26.5 \pm 8.2$ \\
\hline Below cut off value, $n(\%)$ & $1(4)$ & $10(37)$ & $1(10)$ & $6(60)$ & $3(43)$ \\
\hline Weight $(\mathrm{kg})$ & $64.7 \pm 7.1$ & $58.7 \pm 12.9^{*}$ & $60.5 \pm 12.1$ & $58.8 \pm 11.1$ & $56.2 \pm 16.1$ \\
\hline Height $(\mathrm{cm})$ & $161.4 \pm 6.6$ & $163.5 \pm 7.8$ & $163.0 \pm 5.9$ & $163.8 \pm 8.9$ & $163.3 \pm 8.4$ \\
\hline $\operatorname{BMI}\left(\mathrm{kg} / \mathrm{m}^{2}\right)$ & $24.7 \pm 2.2$ & $21.8 \pm 3.6^{* *}$ & $22.4 \pm 3.3$ & $22.2 \pm 3.3$ & $20.4 \pm 3.8$ \\
\hline $\operatorname{ASM}\left(\mathrm{kg} / \mathrm{m}^{2}\right)$ & $7.9 \pm 0.8$ & $7.0 \pm 1.4^{* *}$ & $7.2 \pm 1.2$ & $7.2 \pm 1.4$ & $7.0 \pm 1.6$ \\
\hline Below cut off value, $n(\%)$ & $1(4)$ & $13(48)$ & $5(50)$ & $4(40)$ & $4(57)$ \\
\hline FFMI $\left(\mathrm{kg} / \mathrm{m}^{2}\right)$ & $17.5 \pm 1.7$ & $15.4 \pm 2.5^{* *}$ & $16.2 \pm 2.1$ & $15.7 \pm 2.1$ & $13.8 \pm 2.9$ \\
\hline $\operatorname{SMI}\left(\mathrm{kg} / \mathrm{m}^{2}\right)$ & $7.9 \pm 1.3$ & $6.4 \pm 1.7 * *$ & $7.0 \pm 1.5$ & $6.6 \pm 1.4$ & $5.4 \pm 2.0$ \\
\hline FMI $\left(\mathrm{kg} / \mathrm{m}^{2}\right)$ & $7.2 \pm 2.2$ & $6.4 \pm 2.5$ & $6.3 \pm 1.9$ & $6.4 \pm 3.3$ & $6.6 \pm 2.0$ \\
\hline Gait speed $(\mathrm{m} / \mathrm{s})$ & $2.1 \pm 0.4$ & $1.5 \pm 0.4 * *$ & $1.7 \pm 0.4$ & $1.6 \pm 0.3$ & $1.3 \pm 0.3$ \\
\hline Below cut off value, $n(\%)$ & $0(0)$ & $2(7)$ & $0(0)$ & $0(0)$ & $2(29)$ \\
\hline EAT-10 abnormal, $n(\%)$ & $1(4)$ & $5(19)$ & $1(10)$ & $2(20)$ & $2(28)$ \\
\hline
\end{tabular}

Mean \pm standard deviation

$G O L D$ global initiative for chronic obstructive lung disease, $M T P$ maximum tongue pressure, $B M I$ body mass index, $A S M$ appendicular skeletal muscle mass, FFMI fat free mass index, FMI Fat mass index, EAT-10 eating assessment test-10

$* p$ value $<0.05$ vs control subjects, $* * p$ value $<0.01$ vs control subjects. There were no significant differences among GOLD stages 
Fig. 2 MTP difference between COPD patients and control subjects. The data are presented as mean \pm standard deviation. $M T P$ maximum tongue pressure; $C O P D$ chronic obstructive pulmonary disease. ${ }^{*} p=0.032$

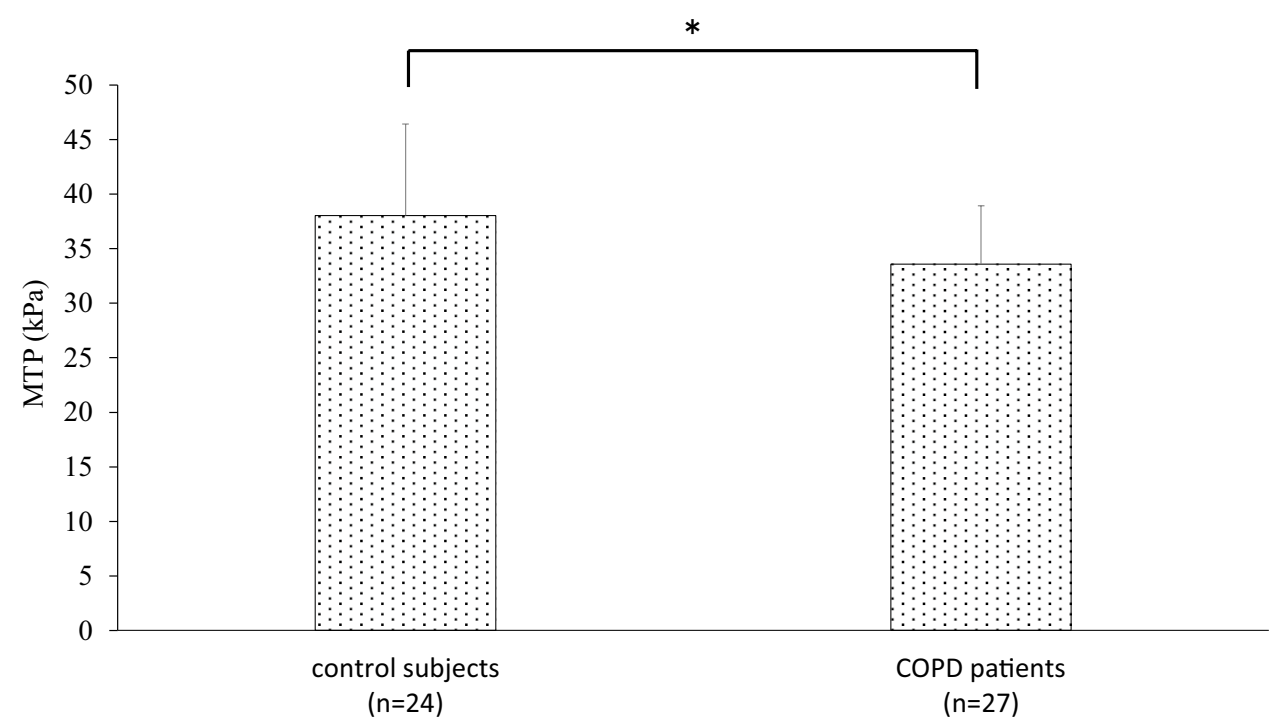

strength, and gait speed were also significantly lower in COPD patients $(p=0.004, p=0.001, p=0.001, p=0.006$, $p<0.001$, respectively). Of the COPD patients, $19 \%$ $(5 / 27)$ were diagnosed with dysphagia, and the prevalence of dysphagia was higher in COPD patients compared to control subjects (1/24). However, the difference was not significant $(p=0.112)$. We could not find any significant difference between each GOLD stage ( $p$ values are shown in Supplementary table 2).

\section{Relationship Between MTP and Other Factors in Each Group}

There was a moderate positive correlation between MTP and SMI in COPD patients ( $r=0.56, p=0.033)$ (Table 3$)$. There was also a moderate positive correlation between MTP and handgrip strength in COPD patients $(r=0.43, p=0.027)$. There was no correlation between MTP and age $(p=0.707)$. In the control subjects, there was no correlation between EAT-10 and sarcopenia-related factors (all measures of muscle and fat free body mass, handgrip strength, and gait speed).

\section{Discussion/Conclusion}

In this study, MTP was significantly lower in patients with COPD compared to control subjects, supporting our hypothesis. In addition, the prevalence of sarcopenia was higher in COPD patients, compared to control subjects. As one of the signs of sarcopenia, MTP was lower along with lower SMI and handgrip strength in COPD patients.

The relationship between sarcopenia and dysphagia can be explained using one of two patterns: one in which
Table 3 Correlation coefficients for MTP with sarcopenia-related factors and pulmonary functions in each group

\begin{tabular}{|c|c|c|c|c|}
\hline & \multicolumn{2}{|c|}{ Control subjects } & \multicolumn{2}{|c|}{ COPD patients } \\
\hline & $\begin{array}{l}\text { Correlation } \\
\text { coefficient } \\
(r)\end{array}$ & $p$ value & $\begin{array}{l}\text { Correlation } \\
\text { coefficient } \\
(r)\end{array}$ & $p$ value \\
\hline Age & -0.11 & 0.626 & -0.08 & 0.707 \\
\hline FVC (L) & $-0.41 *$ & 0.048 & 0.06 & 0.758 \\
\hline$\% \mathrm{FVC}(\%)$ & $-0.42 *$ & 0.039 & -0.19 & 0.333 \\
\hline $\mathrm{FEV}_{1}(\mathrm{~L})$ & -0.37 & 0.073 & 0.13 & 0.489 \\
\hline$\%$ FEV (\%) & -0.34 & 0.105 & -0.16 & 0.937 \\
\hline $\mathrm{FEV}_{1} / \mathrm{FVC}(\%)$ & 0.07 & 0.764 & 0.04 & 0.846 \\
\hline BMI $\left(\mathrm{kg} / \mathrm{m}^{2}\right)$ & 0.06 & 0.774 & $0.52 * *$ & 0.006 \\
\hline $\begin{array}{l}\text { Handgrip strength } \\
\quad(\mathrm{kg})\end{array}$ & -0.24 & 0.259 & $0.43^{*}$ & 0.026 \\
\hline $\operatorname{ASM}\left(\mathrm{kg} / \mathrm{m}^{2}\right)$ & 0.03 & 0.873 & $0.37 *$ & 0.017 \\
\hline FFMI $\left(\mathrm{kg} / \mathrm{m}^{2}\right)$ & 0.05 & 0.811 & $0.50 * *$ & 0.008 \\
\hline SMI $\left(k g / m^{2}\right)$ & -0.02 & 0.918 & $0.56^{* *}$ & 0.003 \\
\hline FMI $\left(\mathrm{kg} / \mathrm{m}^{2}\right)$ & 0.03 & 0.894 & 0.28 & 0.171 \\
\hline Gait speed $(\mathrm{m} / \mathrm{s})$ & -0.04 & 0.895 & 0.29 & 0.144 \\
\hline EAT-10 & -0.30 & 0.156 & 0.22 & 0.264 \\
\hline
\end{tabular}

$F V C$ forced vital capacity, FEVI forced expiratory volume in one second, MTP maximum tongue pressure, $B M I$ body mass index, $A S M$ appendicular skeletal muscle mass, $F F M I$ fat free mass index, SMI: skeletal muscle mass index, FMI fat mass index, EAT-10 eating assessment test-10

${ }^{*} p$ value $<0.05,{ }^{* *} p$ value $<0.01$

whole-body sarcopenia is followed by a swallowing disorder, and one in which a swallowing disorder is followed by malnutrition/whole-body sarcopenia [24]. Muscle dysfunction is a major complication for COPD patients [25]. In the UK and the Netherlands, the prevalence of quadriceps weakness in COPD patients was $32 \%$, and it tended to be 
observed in the early stages of COPD (GOLD stage I + II) [9]. Decreased skeletal muscle mass is a potential risk factor for dysphagia [26]. This suggests that whole-body atrophy may result in weakness of the swallowing muscles in COPD patients. In addition, MTP values are associated with ASM and handgrip strength but not with age. These results suggest that sarcopenic dysphagia is caused by sarcopenia and whole-body atrophy rather than age-related changes.

Putative triggers of muscle wasting in COPD patients include disuse, hypoxemia, malnutrition, inflammation, and the use of synthetic glucocorticoids [27]. These may be a direct consequence of decreased function and pathological remodeling of the diseased lung, e.g., hypoxemia and pulmonary inflammation, or may concern physiological and behavioral mal-adaptations, e.g., disuse and malnutrition. During disease exacerbations, these factors converge and, together with the use of synthetic glucocorticoids as a common medical intervention, may synergize to accelerate muscle wasting. This could be one of the mechanisms of muscle dysfunction, including that of the tongue, in COPD patients.

In a previous study, the mechanism of dysphagia in COPD patients was thought to be impairment of breathing and swallowing coordination [28]. The abnormal timing of swallows within the respiratory cycle occurs due to dyspnea and breathlessness. Swallowing during inspiration (the I-SW pattern) and swallowing immediately followed by inspiration (the SW-I pattern) were observed in COPD patients. Patients with either the I-SW pattern or the SW-I pattern were associated with a higher frequency of exacerbation [29]. A recent study showed the effectiveness of continuous positive pressure ventilation (CPAP) to decrease SW-I frequency and normalize the timing of swallowing [30]. While this discoordination has been well evaluated, tongue strength in COPD patients had yet to be evaluated. Ours is the first report of decreased tongue strength in COPD patients.

Despite the differences in MTP between groups, there was no difference in dysphagia as measured by the EAT-10 test between groups. However, according to a systematic review of dysphagia in chronic pulmonary disease, many previous studies reported a prevalence of dysphagia in COPD patients [31]. Hiraoka et al. showed that lower MTP $(<20 \mathrm{kPa})$ is a risk factor for aspiration pneumonia in amyotrophic lateral sclerosis (ALS) patients [5]. In our study, we only recruited patients who were independent in daily living and had no apparent aspiration symptoms. More relevant assessments of swallowing function may be needed, such as swallowing video fluorography or swallowing video endoscopy.

There are several ways to improve tongue strength. Effortful swallowing training (5 days per week for 4 weeks) improved tongue strength in stroke patients with dysphagia [32]. In a randomized controlled trial of tongue-strengthening exercise, anterior and posterior maximal isometric pressure improved after $4-8$ weeks of training [33]. In COPD patients, the prevalence of sarcopenia was higher in patients with malnutrition [34]. This suggests that nutrition support may prevent sarcopenia in COPD patients. In fact, a recent study showed that maintaining skeletal muscle mass had a positive impact on tongue strength gained through dysphagia rehabilitation [35]. Having a social network involving neighbors and taking part in leisure activities were associated with higher tongue pressures [36]. Further research is needed to investigate the benefits of lifestyle interventions (increasing physical activity) and nutritional supplements to determine whether these interventions can prevent sarcopenia and atrophy of the tongue muscles or not.

There are some limitations to this study. The main limitation was the small number of subjects. This may be the reason the prevalence of dysphagia was not different between groups. However, the number of the enrolled subjects was enough to show a difference in MTP. There were not enough patients to compare MTP with severity of COPD. Another limitation was a lack of objective evaluation for swallowing. We did not perform objective measures of swallowing such as video fluoroscopy or video endoscopy. EAT-10, which we did use, does not provide any objective measures of swallowing function. Further studies are needed to better define the relationship between swallowing function and tongue strength.

In conclusion, tongue strength was lower in COPD patients compared to normal subjects.

Supplementary Information The online version contains supplementary material available at https://doi.org/10.1007/s00455-021-10314-3.

Author Contributions RS, YH and TT made substantial contributions to the literature search. RS, YH, MS made substantial contributions to the data acquisition. RS, YH and YC made substantial contributions to the analysis. RS and $\mathrm{YH}$ made substantial contributions to the manuscript preparation. YH, MS, TT, TK, YC, ON, SA, KF, and YT revised the report critically for important intellectual content. All authors approved the final version to be published and agreed to be accountable for all aspects of the work.

\section{Declarations}

Conflict of interest Dr. Tohda Y. has reported personal fees from Kyorin Pharmaceutical, Co., Ltd., Teijin Pharma Co., Ltd., Kyorin Pharmaceutical Co., Ltd., Meiji Seika Pharma Co., Ltd., Boehringer Ingelheim Co., Ltd., Daiichi Sankyo Co., Ltd., and Astellas, Pearl Therapeutics, Inc., outside the submitted work. The other authors report no conflicts of interest.

\section{References}

1. Good-Fratturelli MD, Curlee RF, Holle JL. Prevalence and nature of dysphagia in VA patients with COPD referred for videofluoroscopic swallow examination. J Commun Disord. 2000;33:93-110. 
2. Yoshida M, Kikutani T, Tsuga K, Utanohara Y, Hayashi R, Akagawa Y. Decreased tongue pressure reflects symptom of dysphagia. Dysphagia. 2006;21:61-5.

3. Shaker R, Cook IJS, Dodds WJ, Hogan WJ. Pressure-flow dynamics of the oral phase of swallowing. Dysphagia. 1988;3:79-84.

4. Nakamori M, Hosomi N, Ishikawa K, Imamura E, Shishido T, Ohshita T, Yoshikawa M, Tsuga K, Wakabayashi S, Maruyama $\mathrm{H}$, Matsumoto M. Prediction of pneumonia in acute stroke patients using tongue pressure measurements. PLoS ONE. 2016;11:1-11.

5. Hiraoka A, Yoshikawa M, Nakamori M, Hosomi N, Nagasaki T, Mori T, Oda M, Maruyama H, Yoshida M, Izumi Y, Matsumoto $\mathrm{M}$, Tsuga K. Maximum tongue pressure is associated with swallowing dysfunction in ALS patients. Dysphagia. 2017;32:542-7.

6. Cruz-Jentoft AJ, Bahat G, Bauer J, Boirie Y, Bruyère O, Cederholm T, Cooper C, Landi F, Rolland Y, Sayer AA, Schneider SM, Sieber CC, Topinkova E, Vandewoude M, Visser M, Zamboni M, Bautmans I, Baeyens JP, Cesari M, Cherubini A, Kanis J, Maggio M, Martin F, Michel JP, Pitkala K, Reginster JY, Rizzoli R, Sánchez-Rodríguez D, Schols J. Sarcopenia: revised European consensus on definition and diagnosis. Age Ageing. 2019;48:16-31.

7. Cao C, Wang R, Wang J, Bunjhoo H, Xu Y, Xiong W. Body mass index and mortality in chronic obstructive pulmonary disease: a meta-analysis. PLoS ONE. 2012;7:e43892.

8. Kim HC, Lee GD, Hwang YS. Skeletal muscle dysfunction in patients with chronic obstructive pulmonary disease. Tuberc Respir Dis. 2010;68:125-39.

9. Seymour JM, Spruit MA, Hopkinson NS, Natanek SA, Man WDC, Jackson A, Gosker HR, Schols AMWJ, Moxham J, Polkey MI, Wouters EFM. The prevalence of quadriceps weakness in COPD and the relationship with disease severity. Eur Respir J. 2010;36:81-8.

10. Strandkvist VJ, Backman H, Röding J, Stridsman C, Lindberg A. Hand grip strength is associated with forced expiratory volume in 1 second among subjects with COPD: report from a populationbased cohort study. Int J COPD. 2016;11:2527-34.

11. Robbins JA, Humpal NS, Banaszynski K, Hind J, Rogus-Pulia N. Age-related differences in pressures generated during isometric presses and swallows by healthy adults. Dysphagia. 2016;31:90-6.

12. Nicosia MA, Hind JA, Roecker EB, Carnes M, Doyle J, Dengel GA, Robbins J. Age effects on the temporal evolution of isometric and swallowing pressure. J Gerontol Ser A. 2000;55:M634-40.

13. Maeda K, Akagi J. Decreased tongue pressure is associated with sarcopenia and sarcopenic dysphagia in the elderly. Dysphagia. 2015;30:80-7.

14. Ogawa N, Mori T, Fujishima I, Wakabayashi H, Itoda M, Kunieda K, Shigematsu T, Nishioka S, Tohara H, Yamada M, Ogawa S. Ultrasonography to measure swallowing muscle mass and quality in older patients with sarcopenic dysphagia. J Am Med Dir Assoc. 2018;19:516-22.

15. Tsuga K, Yoshikawa M, Oue H, Okazaki Y, Tsuchioka H, Maruyama M, Yoshida M, Akagawa Y. Maximal voluntary tongue pressure is decreased in Japanese frail elderly persons. Gerodontology. 2012;29:1078-85.

16. Utanohara Y, Hayashi R, Yoshikawa M, Yoshida M, Tsuga K, Akagawa Y. Standard values of maximum tongue pressure taken using newly developed disposable tongue pressure measurement device. Dysphagia. 2008;23:286-90.

17. Miller MR, Hankinson J, Brusasco V, Burgos F, Casaburi R, Coates A, Crapo R, Enright P, van der Grinten CP, Gustafsson P, Jensen R, Johnson DC, MacIntyre N, McKay R, Navajas D, Pedersen OF, Pellegrino R, Viegi G, Wanger J, Force AET. Standardisation of spirometry. Eur Respir J. 2005;26:319-38.

18. Vogelmeier CF, Criner GJ, Martinez FJ, Anzueto A, Barnes PJ, Bourbeau J, Celli BR, Chen R, Decramer M, Fabbri LM, Frith P,
Halpin DMG, Varela MVL, Nishimura M, Roche N, RodriguezRoisin R, Sin DD, Singh D, Stockley R, Vestbo J, Wedzicha JA, Agustí A. Global strategy for the diagnosis, management, and prevention of chronic obstructive lung disease 2017 report. Am J Respir Crit Care Med. 2017;195:557-82.

19. Takahashi M, Koide K, Suzuki H, Satoh Y, Iwasaki SI. Evaluation of reliability of perioral muscle pressure measurements using a newly developed device with a lip piece. Acta Bioeng Biomech. 2016;18:135-43.

20. Chen LK, Woo J, Assantachai P, Auyeung TW, Chou MY, Iijima K, Jang HC, Kang L, Kim M, Kim S, Kojima T, Kuzuya M, Lee JSW, Lee SY, Lee WJ, Lee Y, Liang CK, Lim JY, Lim WS, Peng LN, Sugimoto K, Tanaka T, Won CW, Yamada M, Zhang T, Akishita M, Arai H. Asian Working Group for Sarcopenia: 2019 consensus update on sarcopenia diagnosis and treatment. J Am Med Dir Assoc. 2020;21:300-307.e302.

21. Kurita N, Wakita T, Kamitani T, Wada O, Mizuno K. SARC-F validation and SARC-F+EBM derivation in musculoskeletal disease: the SPSS-OK Study. J Nutr Health Aging. 2019;23:732-8.

22. Bosy-Westphal A, Jensen B, Braun W, Pourhassan M, Gallagher D, Müller MJ. Quantification of whole-body and segmental skeletal muscle mass using phase-sensitive 8-electrode medical bioelectrical impedance devices. Eur J Clin Nutr. 2017;71:1061-7.

23. Belafsky PC, Mouadeb DA, Rees CJ, Pryor JC, Postma GN, Allen J, Leonard RJ. Validity and reliability of the Eating Assessment Tool (EAT-10). Ann Otol Rhinol Laryngol. 2008;117:919-24.

24. Fujishima I, Fujiu-Kurachi M, Arai H, Hyodo M, Kagaya H, Maeda K, Mori T, Nishioka S, Oshima F, Ogawa S, Ueda K, Umezaki T, Wakabayashi H, Yamawaki M, Yoshimura Y. Sarcopenia and dysphagia: position paper by four professional organizations. Geriatr Gerontol Int. 2019;19:91-7.

25. Barreiro E, Bustamante V, Cejudo P, Gáldiz JB, Gea J, de Lucas P, Martínez-Llorens J, Ortega F, Puente-Maestu L, Roca J, Rodríguez González-Moro JM. Guidelines for the evaluation and treatment of muscle dysfunction in patients with chronic obstructive pulmonary disease. Arch Bronconeumol (English Edition). 2015;51:384-95.

26. Maeda K, Takaki M, Akagi J. Decreased skeletal muscle mass and risk factors of sarcopenic dysphagia: a prospective observational cohort study. J Gerontol Ser A. 2017;72:1290-4.

27. Langen RCJ, Gosker HR, Remels AHV, Schols AMWJ. Triggers and mechanisms of skeletal muscle wasting in chronic obstructive pulmonary disease. Int J Biochem Cell Biol. 2013;45:2245-56.

28. Gross RD, Atwood CW, Ross SB, Olszewski JW, Eichhorn KA. The coordination of breathing and swallowing in chronic obstructive pulmonary disease. Am J Respir Crit Care Med. 2009;179:559-65.

29. Nagami S, Oku Y, Yagi N, Sato S, Uozumi R, Morita S, Yamagata Y, Kayashita J, Tanimura K, Sato A, Takahashi R, Muro S. Breathing-swallowing discoordination is associated with frequent exacerbations of COPD. BMJ Open Respir Res. 2017;4:1-9.

30. Hori R, Ishida R, Isaka M, Nakamura T, Oku Y. Effects of noninvasive ventilation on the coordination between breathing and swallowing in patients with chronic obstructive pulmonary disease. Int J COPD. 2019; 14:1485-94.

31. Ghannouchi I, Speyer R, Doma K, Cordier R, Verin E. Swallowing function and chronic respiratory diseases: systematic review. Respir Med. 2016;117:54-64.

32. Park HS, Oh DH, Yoon T, Park JS. Effect of effortful swallowing training on tongue strength and oropharyngeal swallowing function in stroke patients with dysphagia: a double-blind, randomized controlled trial. Int J Lang Commun Disord. 2019;54:479-84.

33. Van den Steen L, Vanderwegen J, Guns C, Elen R, De Bodt M, Van Nuffelen G. Tongue-strengthening exercises in healthy older adults: does exercise load matter? A randomized controlled trial. Dysphagia. 2019;34:315-24. 
34. de Blasio F, Di Gregorio A, de Blasio F, Bianco A, Bellofiore B, Scalfi L. Malnutrition and sarcopenia assessment in patients with chronic obstructive pulmonary disease according to international diagnostic criteria, and evaluation of raw BIA variables. Respir Med. 2018;134:1-5.

35. Nakazawa Y, Kikutani T, Igarashi K, Yajima Y, Tamura F. Associations between tongue strength and skeletal muscle mass under dysphagia rehabilitation for geriatric out patients. J Prosthodont Res. 2020;64:188-92.

36. Nagayoshi M, Higashi M, Takamura N, Tamai M, Koyamatsu J, Yamanashi H, Kadota K, Sato S, Kawashiri SY, Koyama Z, Saito T, Maeda T. Social networks, leisure activities and maximum tongue pressure: cross-sectional associations in the Nagasaki Islands Study. BMJ Open. 2017;7:1-10.

Publisher's Note Springer Nature remains neutral with regard to jurisdictional claims in published maps and institutional affiliations.
Yuji Higashimoto $\mathrm{MD}, \mathrm{PhD}$

Masashi Shiraishi PT, MSc

Tomomi Tamura SLP

Tamotsu Kimura PT

Yasutaka Chiba $\mathrm{PhD}$

Osamu Nishiyama MD, PhD

Shinichi Arizono PT, PhD

Kanji Fukuda MD, PhD

Yuji Tohda MD, PhD

Ryuji Sugiya PT, MSc 